\title{
THE BEST LAID PLANS: COMPUTATIONAL PRINCIPLES OF ACC
}

\author{
Clay B. Holroyd* \\ Tom Verguts \\ Department of Experimental Psychology \\ Ghent University \\ Henri Dunantlaan 2 \\ 9000 Gent, Belgium
}

*Correspondence: clay.holroyd@ugent.be (C.B. Holroyd)

\section{Keywords:}

anterior cingulate cortex

computational models

artificial intelligence

hierarchical model-based hierarchical reinforcement learning

distributed representations

cognitive control

\begin{abstract}
Despite continual debate for the past thirty years about the function of anterior cingulate cortex (ACC), its key contribution to neurocognition remains unknown. However, recent computational modelling work has provided insight into this question. Here we review computational models that illustrate three core principles of ACC function, related to hierarchy, world models and cost. We also discuss four constraints on the neural implementation of these principles, related to modularity, binding, encoding and learning and regulation. These observations suggest a role for ACC in hierarchical model-based hierarchical reinforcement learning, which instantiates a mechanism for motivating the execution of high-level plans.
\end{abstract}




\section{The Riddle of Anterior Cingulate Cortex: Computational Clues}

Humans enjoy a myriad of cognitive abilities that robots, if they could dream, would only dream of. Simply arranging a summer camping trip entails setting goals, planning over multiple time scales (minutes, hours, days, even years), sequentially executing the actions in the plan, monitoring their progress, and staying on course despite challenges along the way. How do we do this?

One brain structure that is likely key for this process is the anterior cingulate cortex (ACC), an extensive tract of neural territory lying along frontal midline cortex (though definitions differ; see Box 1). Despite controversy about what it actually does [1], formal computational models have provided substantial insight into its function $[2,3]$. Here we review three core computational principles underlying ACC: hierarchical decision making, spatio-temporal models of the environment, and the evaluation of a control-related cost. Using the language of computational reinforcement learning (RL) (see Glossary), we propose that ACC contributes to hierarchical model-based hierarchical reinforcement learning (HMB-HRL) (also called "saltatory model-based HRL"; [4]). We then discuss four neural constraints on the physical realization of this abstract function: modularity, binding, encoding, and learning and regulation. These observations elucidate the role of ACC in motivating the execution of high-level plans.

\section{Hierarchical decision making}

Hierarchy provides several advantages for biological and artificial agents alike. First, hierarchical representations can dramatically reduce the size of otherwise intractably-large state and action spaces. Based on the applause of an audience, for example, a pianist might learn which piece they should play (a high-level problem) rather than how to sequence each of the individual keystrokes within the piece (a low-level problem). Second, hierarchical structure minimizes crosstalk between modules: The keystrokes between different pieces are less likely to interfere with each other if they are encoded separately. And third, much like calling a subroutine in a computer programming language, hierarchy enables the recycling of solutions to subparts of a problem. Thus, a musical refrain can be recycled within and across songs. These advantages are leveraged in hierarchical reinforcement learning (HRL), an RL approach that groups together collections of meaningfully-related low-level actions into units 
(called "options") that can be manipulated at higher levels of temporal abstraction [5]. An agent can then learn the value (expected reward) for each option according to standard RL techniques and use those values to select the options.

Although early theories [6] and models [7] of ACC naturally distinguished between higher-level systems that do the controlling and lower-level systems that are controlled, they did not address reward-based action or option selection. Conversely, although it was concurrently recognized that ACC contributes to reward-based action selection [8], there were few early attempts to formalize this idea and generalize it to options. One exception was a model that proposed that ACC uses rewards to learn which task strategy (or option) to apply, whereas other (lower-level) systems learn the policy for each strategy [9] (see also [10]). In line with this framework, recent ensemble neural recordings in rats [11], single-cell recording and electrical microstimulation studies in monkeys [12] and studies with human fMRI [13] have indicated that ACC uses rewards to learn the value of task strategies and switch between them adaptively. Moreover, the discriminability of task representations in ACC following switches (see the Encoding section below) scales inversely with task switch costs [14].

A more recent HRL model of ACC (HRL-ACC) exploited hierarchy by simulating the behavior of rats (which express a homolog of the primate ACC; Box 1), with and without ACC lesions [15]. This model learned the values of different options (whether to navigate using an egocentric or allocentric frame of reference), where each option corresponded to sequences of low-level movements in the maze. This conceptualization aligns well with increasing evidence that ACC is sensitive to hierarchically-organized action representations $[16]$ and reward signals $[17,18]$. It is also consistent with observations that ACC supports foraging behavior [19], which is naturally framed as a hierarchical problem.

These considerations suggest that ACC utilizes rewards to learn appropriate high-level actions (options) according to HRL principles.

\section{Models of the environment}


The term "model" refers to an explicit list of transitions between environmental states that an agent can use for planning flexible, goal-driven behavior (not to be confused with "computational models"). For example, such a model could store information that, on a camping trip, one can travel from the lake to the trailhead or to a favorite berry patch. Whereas canonical model-free reinforcement learning (MF-RL) algorithms associate individual states and actions with learned cached values that allow for relatively inflexible behavior, of which the Rescorla-Wagner algorithm is the most well-known member of the class, algorithms that use models of state transitions can be used for planning to produce flexible, goal-driven behavior [5]. Thus, when a goal rapidly switches from hiking out of the forest to foraging for berries, model-based reinforcement learning (MB-RL) can be used immediately to predict the location of the nearest berry patch.

Growing evidence indicates that ACC, together with other brain areas [20], contributes to MB-RL. For example, a prominent computational model of ACC contains units that exhaustively predict all possible states of the task environment [21] (see also [22]). Such representations could be leveraged to predict target trajectories, as observed in the firing rates of monkey ACC neurons during a pursuit task [23], and to determine the distance between the current state and the goal state, as found across a variety of human fMRI studies of goal-directed behavior [24]. Further, fMRI studies indicate that model-based decision making is associated with ACC activation [25], and that ACC prediction errors [26] correlate with (and presumably trigger) offline replay of hippocampal activity that, in turn, facilitates modelbuilding in ACC [27]. ACC activity is also associated with the prospective value of action plans [28]; with the depth of model-based planning [29]; with increased computational demands at initial choice points and immediately before errors during plan execution [30]; and with the re-evaluation [27] and abandonment [31] of plans. Moreover, ensemble activity in rat ACC is thought to form a topographicallyorganized representational space for the "scaffolding" of behaviorally relevant events; these representations sometimes encode the locations of distal rewards for a prospective purpose [32]. Damage to rat ACC therefore causally disrupts model-based RL [33].

These considerations suggest that ACC plans and executes extended behaviors based on learned world models. 


\section{Evaluation of a control-related cost}

Unlike the principles of hierarchy and world models, a cost principle was introduced into ACC models primarily for empirical rather than computational reasons. Empirically, the deployment of high-level control over task-execution appears to incur a cost that, phenomenologically, is experienced as effortful and is therefore aversive, and hence is minimized according to a cost-benefit tradeoff [34]. Such a cost was implicit in the seminal response conflict model of ACC, which incorporated a self-regulating control mechanism that increased control only when it was needed [7]. More recent models of ACC have explicitly accounted for the cost of control in regulating effortful behaviors (e.g., [35,36]). For example, in line with recent behavioral evidence [37], the HRL model of ACC regulates control at multiple levels of hierarchy, disengaging when control is not required to maintain a high average reward rate [15].

Yet despite the empirical evidence, this principle provides no obvious benefit to the agent: selfregulating control would appear to be both computationally [38] and evolutionarily [39] maladaptive. Accordingly, it is unclear whether the cost of control is a "bug" or a "feature" of the system. On one side, the bug view proposes that the cost reflects biophysical constraints. For example, self-control could consume a limited glucose supply, though this hypothesis has been vigorously disputed [40]. The exercise of control could also temporarily deplete neurotransmitter levels such as dopamine [36], and/or contribute to the buildup in the brain of toxic waste products like amyloid-beta [38]. In these cases, the relaxation of control would prevent against these negative outcomes.

Alternatively, the feature view proposes computational constraints to control. For example, it has been argued that to accommodate opportunity costs incurred when the value of an alternative task exceeds that of the current task, the relaxation of control facilitates a task switch [41]. However, there is no obvious reason why opportunity costs should impair task performance before the switch. Likewise, capacity constraints associated with difficult neural computations, such as interfering task representations [42], do not address why control over any single task should vary over time. Another possibility is that, when control is not required because the task is very easy, control can actually impair performance by slowing it down [43]. However, this explanation does not address why control sometimes wanes on difficult tasks wherein control is always advantageous. 
Taken together, these considerations indicate that ACC self-regulates control levels in order to minimize a concomitant cost, the source of which remains a vexing question in cognitive neuroscience.

\section{Neural Implementation}

A complete theory of ACC function must ultimately address the neural organization of its computational properties. Here we address this issue by asking four questions relevant to the implementation of the three principles above. First, to what extent is ACC function organized into separate modules, such that different parts of ACC carry out different levels of hierarchical model-based planning? Second, how are these computations bound together across space and time? Third, how is each function encoded within local networks of neurons? And fourth, how are the computations learned and regulated?

\section{Modularity}

Planning and decision making across hierarchical levels could be facilitated by modular structure whereby localized neural communities compute functions that are unique to their group. Consistently, frontal cortex appears to be organized modularly, such that more rostral parts of frontal cortex are responsible for increasingly abstract or superordinate computations [44]. This proposition has been extended to ACC, where rostral (rACC) relative to dorsal ACC (dACC) (Box 1) is presumably involved in higher-order aspects of task organization [45]. For example, the hierarchical error representation (HER) model proposes that dACC computes prediction errors about specific events, whereas rACC computes prediction errors related to the task context in which those events occur [21]. The HRL-ACC model applies a similar division of labor to the domain of action selection: dorsal areas of ACC, which are closely linked to motor behavior, control performance over specific tasks (such as climbing a barrier in a maze), whereas rostral areas of ACC control the execution of higher-level options (such as switching between egocentric and allocentric navigation strategies) [15]. Recent support for this idea comes from fMRI studies revealing that $\mathrm{dACC}$ is sensitive to reward values corresponding to overt gains, whereas rACC is more sensitive to higher-level goals [46]; and that dACC accumulates evidence about how best to respond in a task, whereas rACC accumulates evidence about what task strategy to deploy [47]. Further, a recent study that recorded the intracranial electroencephalogram in humans revealed that 
rACC determines the set point at which the agent switches between action plans, and dACC utilizes feedback to determine whether to continue with or switch between the plans according to that set point [48].

\section{Binding}

In modular systems, computations must be bound both across space (modules) and across time. Oscillations are a good candidate for this purpose because they can link different modules involved in the same cognitive process [49], and can organize the execution of action sequences into useful hierarchies across time [50]. In the brain, such oscillations are thought to be implemented via interactions between excitatory (pyramidal) and inhibitory (inter)neurons, which can be observed intracranially in the local field potential and in the extracranial electroencephalogram [51].

Neural oscillations thereby provide a means for rACC and AACC to coordinate their planning activities both with one another and with other brain areas in order to carry out extended, goal-directed sequences over time. A series of models $[10,52]$ propose that ACC orchestrates this binding between perceptual and motor modules. Specifically, when task demands are high (e.g., after an error), ACC would send a synchronizing signal to lower-order modules, with consequent synchronization and thus improved communication between those lower-order modules. In line with a wealth of neural data, it was proposed that low-level controllers can phase-lock in the gamma frequency band and consequently communicate more efficiently [49]. Slower theta frequency oscillations, which have been measured prominently in medial frontal cortex [53], would be used to achieve this phase-locking of the correct modules.

Flexible integration and segregation of these modules could be facilitated by subcortical systems that release neuromodulators such as norepinephrine and acetylcholine in cortex [54]. For example, phasic bursts of norepinephrine, which may serve as a neural-interrupt signal [55], can reset network activity in ACC [56] and thus allow for module re-binding.

\section{Encoding}

Although HRL reduces the size of the state and action search space in standard RL, this space can still 
be vast. This challenge can be further ameliorated with the use of function approximation, which provides a means to generalize across states or actions that share similar features. In practice, artificial neural networks (ANNs) trained for this purpose produce distributed representations across the activation states of their internal units (Box 2). As a result, the states and actions encoded by the network cannot be inferred from the activity of any single or representative unit, but must be derived from a global analysis of the neural population [57].

In line with this, it is increasingly recognized that neural representations are distributed [58]. This may be especially true of ACC and other frontal brain areas [59]. Individual ACC neurons seem capable of responding to most task events, with particular mixtures of sensitivities within and across neurons continually reallocated according to changing task conditions [6o]. It follows that standard, univariate statistical approaches that analyze the average activity of populations of neurons (or, in fMRI, the average BOLD response across groups of contiguous voxels) can overlook important information encoded in ACC. For example, multivariate statistical summaries of ensemble ACC activity in rats [61] (Fig 1a), monkeys [62] (Fig 1b, left) and humans [63] (Fig 1c, left) highlight a role for ACC in tracking the progress of the agent through sequences of goal-directed actions-properties that would be difficult to infer from the response profiles of individual neurons or voxels. It is also noteworthy that both the presence of response conflict [64] and the administration of amphetamine (a dopaminergic agonist) [61] enhance the discriminability between task representations, illustrating how changing control levels can impact the course of the trajectories.

Recent models of ACC have used ANNs to provide insight into these representations. One computational model of ACC function simulated the steps in a sequence production task using a recurrent neural network (RNN) [22]. When the internal states of the network were assessed with multivariate statistics, the results were broadly consistent with the ensemble activity of ACC neurons in rats performing the same task. Inspired by previous simulations (Box 2), this model was later applied to predict distributed patterns of ACC activity as humans performed a sequential task (Fig 1C, right), which was then tested and confirmed with fMRI (Fig 1c, left) [63]: model and data reveal cyclic patterns of activity underlying sequence execution that generalize across the same action categories. Similarly, Fig. $1 \mathrm{~b}$ (right) shows the dynamics of the internal representation (measured at the hidden units) of an RNN 
trained on the steps of an RL task, incorporating a "context" neuron that separates a beginning trialand-error learning phase from a later applied phase of the task. The state space splits into two trajectories corresponding to the two phases (red vs. blue lines), forming a type of hierarchical representation of task execution similar to the patterns observed in ACC of monkeys engaged in the same task (Fig 1b, left) [62]. And although not about ACC per se, Fig 1d illustrates a heteroclinical channel, a mathematical object derived from non-linear dynamical systems theory [65-67], which represents hierarchical action sequences as successive metastable states [68]. The trajectories of the channel describe 3 hierarchically organized action sequences: blue trajectories illustrate the dynamics within each sequence, and green trajectories illustrate the dynamics when switching between sequences. These equations predict relatively slower changes within sequences and faster changes between sequences, as observed in rat ACC (Fig 1a, where arrow length indicates the speed of the trajectory), and separation between sequences, as observed in monkey ACC (Fig 1b, left, blue vs. red lines). A recent study applying dynamical systems theory to neighboring brain areas [69] exemplifies how the approach might be similarly applied to ACC.

\section{Learning and Regulation}

How are these planning-related processes learned, and once learned, how are they regulated during their execution? This is not an easy computational problem. First, ACC must learn how to parcellate action hierarchies and world models into modules that accord with statistical regularities in the environment [70]. Although there is reason to believe that ACC learns such hierarchical structure according to RL principles, the underlying algorithms are still under active scrutiny (Box 3). Second, RL models have historically represented hierarchical structure in modular or tabular form. In contrast, as described above, ACC appears to encode hierarchical structure with distributed representations, which makes the parcellation problem even harder. Third, these representations can be learned through algorithms that follow a slow, iterative process of gradient descent [71], but the existence of a neural analog for this process has been debated for decades [72]. And fourth, it is an open question how slow weight changes associated with rule learning in ANNs could give rise to fast reconfigurations of network-wide activity observed in ACC (and other brain areas), such as abrupt transitions when rats switch between task contexts and sequences [6o]. 
Although much remains unknown, the impact of neuromodulators on cortical function is likely to play a key role in the resolution of these problems. For example, reward prediction errors that drive RL in many ACC models could be carried to ACC via midbrain dopamine neurons [9]. Strikingly, a recent computational model shows how dopamine-dependent reward prediction errors can slowly train RNNs in prefrontal cortex to compute task-related functions, which in turn can rapidly learn new rules by autonomously updating their own activation-based representations [73]. These efforts point to biologically plausible mechanisms for accelerating the learning of new representations in ACC and other brain areas [74].

In parallel, the tonic firing of dopamine neurons can stabilize effort-related neural representations in ACC against disruptions [75]. In the HRL-ACC model, for example, a rat's persistence at achieving a goal (climbing a high barrier to get a large reward) is supported by an average reward signal mediated by tonic dopamine levels in ACC [15]. Conversely, a dopamine-dependent average reward signal could facilitate the switch to a different task-that is, to desist on the current task-according to its opportunity cost $[76]$.

\section{Integrating ACC Principles and Constraints}

Although other brain areas contribute to hierarchy, models, and the cost of control, we propose that ACC occupies a privileged position at the intersection of all three (Fig 2, Key Figure). This arrangement endows it with a unique computational function. First, by combining hierarchical representations with world models, ACC can use HMB-HRL to evaluate action sequences that have especially long temporal horizons [4]. This ability is afforded by a modular structure whereby more rostral areas of ACC (and neighboring brain areas) implement schemas-high-level abstract knowledge structures that organize low-level representations that guide behavior [77,78]-and relatively caudal areas of ACC contribute more directly to action production. This enables ACC to plan not only over sequences of individual actions (such as the steps along one trail or another), but also over chains of entire sequences (such as going to the next fork in the path, taking it to a lake, unpacking the tent, and so on) [79]. In keeping with this architecture, fMRI evidence indicates that parts of ACC encode the higher-level as opposed to 
lower-level actions, e.g., the trail taken rather than the individual steps along the trail [16].

Second, once put into execution, ACC can evaluate the projected reward value of the plan (as given by the world model) against its ongoing control-related cost. This function has outsized importance given that the high computational demands of planning often allow for only approximate results [80]: People typically initiate plans without knowing the specific paths that take them from one subgoal to the next [81], leaving the low-level system to work out the details on-the-fly [79]. Based on an ability to generalize afforded by its distributed encoding scheme, ACC could match the current state to a suitable plan-a critical function when the projected rewards of a plan approach its accruing costs, which can tip the motivational balance in favor or against plan completion. As a consequence, high control levels ensure that the low-level system figures out how to implement the plan despite the control-related cost of doing so. In fact, a wealth of evidence $[19,53,82]$ converges on the idea that the ACC is responsible for sticking to a plan, i.e., for persevering [15,45]; and conversely, damage to ACC attenuates goaldirected behavior, resulting in apathy $[45,53,82]$.

A key question is whether the learned models are stored in ACC or are simply sent to ACC for processing. Whereas the hippocampus is likely involved in the construction of world models [83], it seems less directly involved in evaluating the costs and benefits of plans derived from those models. Rather, ACC appears to work with the hippocampus to rapidly chain together episodic memories of sequential events [84] in order to prospectively identify goal-directed action sequences [32], a process mediated by binding the modules with oscillations. In line with this supposition, temporary disturbance of ACC activity impairs theta-band communication with the hippocampus, thereby disrupting episodic future thinking that underlies the initiation of deliberative action sequences [85]. Further, although the hippocampus can develop abstract representations of task elements that occur in different environments [86], it appears less able to abstract higher action and goal structure from multiple encounters with different tasks, which would yield compositional representations that afford cognitive flexibility [87]. Instead, such representations align more closely with rACC, which is sensitive to task states [88], action schemas [77,78], and world models for multi-step action plans [83]. This suggests that the ACC learns and stores hierarchical models for subsequent execution, beyond simply evaluating information passed to it from other brain areas. 
Taken together, these principles and constraints suggest an intriguing answer to the question about the origin of the cost of control: the exercise of control by ACC could preclude it from learning new hierarchical representations, due to mutual interference between the control and learning processes (Box 4).

\section{Concluding Remarks}

Even the best laid plans go awry. We have argued that ACC evaluates and selects plans, including intervening when ongoing events take a turn for the worse. Imagine that a freak snow storm looms while on a camping trip. Should we risk getting trapped in the snow by following a planned, slow route out of the wilderness? Or should we navigate a faster but more treacherous passage through unknown territory? Both of these scenarios might correspond poorly with our pre-existing world models and options. It would be the responsibility of ACC to find existing hierarchical representations (or schemas) that best fit the situation, derive novel plans from them, evaluate their potential, and ultimately carry out the best plan despite the unknowns. On the data reviewed here, all of this is guided by ACC using HMB-HRL under the influence of a limited control signal that fatigues with overuse. Although a full understanding of this mechanism awaits further collaboration between empirical and computational scientists (see Box 3 and Outstanding Questions), the answer is on horizon, if we stay the course. 


\section{BOXES}

\section{BOX 1: MULTIPLE DEFINITIONS OF ACC}

Controversy over anterior cingulate cortex (ACC) extends even to its nomenclature. The functional neuroimaging literature commonly equates ACC with the ventral aspect of the entire frontal midline cortex, as illustrated by a probabilistic map of ACC (yellow) and adjacent areas (green) generated from a brain image database (Fig IA) [89]. As classically conceived, ACC is bounded within frontal medial cortex by the corpus callosum ventrally and by the cingulate sulcus or paracingulate sulcus dorsally. This region is separated at the level of the genu of the corpus callosum (vertical white line) into rostral (rACC) and dorsal and caudal (dACC) parts. Also shown are the pre-supplementary motor area (pre-SMA) and medial frontal gyrus (MFG).

An alternative schematic of cingulate cortex by Vogt, based mainly on neuroanatomical considerations, classifies cingulate cortex into ACC, posterior cingulate cortex (PCC), and midcingulate cortex (MCC) regions; the latter is further subdivided into anterior (aMCC) and posterior (pMCC) areas (Fig IB). In this review we adopt the more common usage of rACC and AACC, which correspond approximately to the regions ACC and aMCC as defined by Vogt.

A parallel debate has questioned the existence of ACC homologs, including whether rodents even have a prefrontal cortex. Recent considerations $[90,91]$ indicate that regions of rat frontal midline cortex (colored circles, Fig IC, left) corresponding to cingulate cortex (Cg, area 24, blue), prelimbic cortex (PL, area 32, red), and infralimbic cortex (IL, area 25, purple) (Fig IC, middle) constitute homologs of the human aMCC, posterior (pACC) and subgenual (sACC) ACC (Fig IC, right), as defined according to the Vogt nomenclature.

Figure I. NEUROANATOMY OF ANTERIOR CINGULATE CORTEX. Fig IA adapted from [89] with permission. Fig IB adapted from [92] with permission. Fig IC adapted from [90] with permission. 


\section{BOX 2: DISTRIBUTED REPRESENTATIONS AND NEURAL NETWORKS}

Distributed representations contain information that is not reducible to single states or actions; instead, state and action representations depend on multivariate patterns of activity across the population [93]. Used in this sense, the term should not be confused with networks of moduleswhich are also distributed, as discussed in the section on binding-but rather networks of neurons within a module, i.e., neurons that are concerned with the same computational function [65]. In practice, the analysis of distributed representations in biological neural networks typically requires the application of dimensionality reduction techniques that characterize the ensemble activity in terms of a set of underlying factors [94]. For networks that change with time, these patterns can be described with phase portraits wherein each dimension corresponds to one of these factors and the temporal evolution of the network is characterized as a trajectory through that state space [58,65-67].

It has long been recognized that insight into distributed representations in neural systems can be drawn from artificial neural networks (ANNs) $[57,67,95]$. Standard learning procedures used to train ANNs yield, as a consequence of learning, distributed representations across the activation states of the internal network units. These representations often reveal essential properties of ANNs, such as the abilities for pattern completion and function approximation that facilitate generalization across exemplars. For example, training a recurrent neural network (RNN) across multiple tasks can sometimes yield internal representations that reflect compositionality of task representations [87], thereby providing means to optimize the performance of the control system across tasks [42].

Likewise, an RNN (Figure I, left) trained to carry out sequences of steps in the sub-task "adding sugar" in the context of the tasks "making coffee" vs. "making tea" yields trajectories in state space that evolve in parallel (Figure I, right: solid red line and dashed blues line correspond to coffee and tea tasks, respectively; circles and crosses indicate start and end states, respectively). These trajectories indicate shared structure between the internal representations for the two tasks (due to generalization by the network between making tea and making coffee), but do not coincide exactly (reflecting the maintenance of contextual information necessary to distinguish between tasks) [96]. These observations suggest that inspection of distributed representations in real neural systems tasked with analogous functions could reveal similar properties [58].

Figure I. EXAMPLE RECURRENT NEURAL NETWORK AND STATE SPACE TRAJECTORIES. Adapted from [96] with permission. 


\section{BOX 3: ACC: AT THE CONFLUENCE OF NEUROSCIENCE AND AI}

Neuroscience and artificial intelligence (AI) have long benefitted from mutual cross-fertilization [97] spanning the domains of control theory [98], symbolic reasoning [57], neural networks [57, 95], reinforcement learning (RL) [5], and more. Yet despite high-level control being a central organizing principle in cognitive neuroscience [99], most AI algorithms do not regulate behavior at higher degrees of granularity. Indeed, hierarchically-organized flexible behavior, which exploits compositional structure that can make task processing and generalization more efficient [100], continues to be "perhaps the hardest challenge for AI research" [97]. For this reason, it has been proposed that the next major advances in neuroscience and AI will center on the high-level control of behavior [101-103].

These advances will necessitate the development of algorithms that describe world models that lack full detail, that can be composed at different levels of abstraction, and that can be combined on-the-fly [104], as well as be sensitive to computational costs-all key properties associated with ACC (see main text). Yet the search for the specific algorithm underlying ACC function is complicated by the fact that such models can decompose into multiple kinds of abstract representations and hierarchies [70]. Moreover, in biological systems even the distinction between model-free and model-based RL has been questioned [105].

Current advances in AI serve up a smorgasbord of control-related algorithms that ACC might incorporate. For example, the celebrated game-playing program AlphaGo Zero, recently shown to achieve superhuman performance on the games Go, shogi and chess without any prior domain knowledge, combines model-based search with model-free RL [106]. Alternatively, "successor representations" (SRs)-model-like structures that predict how often an agent will visit any state of the environment starting from any other state-facilitate goal-directed behavior without incurring the costs of model-based planning, while also enabling option discovery by identifying bottleneck states in the state transitions [107]. Further, a recent hierarchical formalism using SRs allows for fast deployment of learned action policies on new tasks [108]. A different approach utilizes eigenoptions to decompose task structure into options that span multiple time scales, which can yield better task performance than options derived from bottleneck states [109]. And interestingly, the introduction of a cost when switching between options (not unlike the switch costs assumed in models of cognitive control, e.g., [15]) can also facilitate the construction of new options [110]. Lastly, a recent biologically plausible computational model describes a flexible planning mechanism based on oscillations and associative learning principles [111]. 


\section{BOX 4: THE ORIGIN OF THE COST OF CONTROL?}

A persistent question in cognitive neuroscience concerns the cost of control [34]. Current debates surround whether this cost reflects a "bug" (a biological constraint) or a "feature" (a computational benefit) of the control system. An intermediate possibility is that the cost reflects a computational solution to a biological constraint, namely, that the biological implementation of control precludes learning new hierarchical representations. On this view, the cost solves a conflict between short-term vs. long-term neural mechanisms for behavioral adaptation by deliberately interleaving periods of high and low control.

ACC may apply control by synchronizing the computations of disparate brain modules using theta oscillations $[10,52]$. Yet the transfer and consolidation of new memories from hippocampus to cortex appears to depend on hippocampal sharp wave-ripples (SWRs), which reflect faster, synchronous neural firing [112]. These two processes could be mutually exclusive. If flexible control requires developing abstract representations across tasks (Box 3), then the application of control could preclude their construction. Notably, sleep allows for the replay of hippocampally-dependent episodic memories that enable their consolidation in cortex [113], which recent RL algorithms (like DYNA [5]) mimic by alternating periods of task performance with periods of "sleep" that promote consolidation. This learning mechanism could extend to the waking state, where the intermittent relaxation of control-even during task performance-would permit ACC to construct abstract, hierarchical models from hippocampally-encoded episodes.

Suggestive of this, pauses between tasks-not just sleep-facilitate memory consolidation in humans [114]. Neuroimaging studies reveal that previously-encoded sensory representations are spontaneously reactivated in cortex during awake rest periods [115,116]. Moreover, reactivation of rest period hippocampal representations correlate with medial frontal representations during task performance, which suggests that the hippocampus trains these cortical representations [117]. This occurs during periods of awake SWRs, when medial frontal representations are suppressed to promote transfer of hippocampal representations [118].

The reduction of control by ACC during waking states could therefore promote learning of task representations underlying world models for planning. Thus, when rats are held immobile (and control is presumably minimized), rostral ACC replays sequential behaviors that occur in a ruleswitching task [119]. These dynamics shift from an early learning period when reverse-replay of action sequences encode new memories in the hippocampus to a later period when forward replay of these sequences are used by medial frontal cortex for planning [120]. Consistent with this, human fMRI indicates that off-task replay of task-related activity in ACC facilitates model building and planning [38]. 


\section{GLOSSARY:}

Artificial neural network: A computer model consisting of neuron-like units that are connected via artificial dendrites and axons. Activation is passed via these connections; learning consists of changing the strengths of the connections.

Bottleneck state: A state that separates regions of state space, for example, a doorway that separates different rooms. An agent must pass through the bottleneck in order to travel from one region to the other.

Cached values: Across-time averaged reward associated with specific stimuli or actions. Learning cached values is time-costly but places minimal burden on the cognitive agent during action execution.

DYNA: Reinforcement learning architecture where a model-free system defines the policy, and a model-based system trains the model-free system. Training typically occurs during periods where the model-free system is offline.

Eigenoption: An option that traverses a principal direction of a learned representation of the environment, which is discovered by following an intrinsic reward function that encourages exploration. Eigenoptions operate at different time scales and can be easily sequenced.

Hierarchical model-based (HMB) hierarchical reinforcement learning (HRL): HRL abstracts across the action space via an option space, where each option combines a set of (primitive) actions. For example, on a road trip "Go Left" might be an action; "Go to Ghent" might be an option. HMB abstracts across the stimulus or state space in a similar manner. For example, on the same road trip, specific locations in a village may constitute the states; and the villages (or even regions of villages) the higher-order states across which HMB plans are made.

Metastable state: As used in neuroscience, a semi-transient neural state that persists for a period of time outside the system's natural equilibrium state.

Model-based reinforcement learning: Acting and learning in order to maximize reinforcement, using a model of the world (i.e., a model of the state transitions in the world).

Model-free reinforcement learning: Acting and learning in order to maximize reinforcement, without a model of the world. This approach uses cached values.

Modules: As used in cognitive neuroscience, localized neural communities that compute functions that are unique to their group.

Non-linear dynamical system: A system of elements (e.g., neurons) that evolves over time according to non-linear differential equations.

Policy: An algorithm that specifies an agent's behavior for any state of the environment. 
Prediction error: Difference between an event (e.g., reward delivery) and a prediction of that event (e.g., a prediction of reward).

Recurrent neural network: An artificial neural network with feedback connections.

Reinforcement learning: Acting and learning in order to maximize reinforcement.

Rescorla-Wagner algorithm: One of the simplest error-drive learning rules. Its generalization toward multi-layer models is the backpropagation algorithm.

Response conflict: Quantification of the extent to which two or more task-incompatible responses are simultaneously active.

Sharp wave-ripple: Short-time, high-frequency neural oscillations associated with memory retrieval and consolidation.

Switch costs: The delay in response times when switching to a new task compared to repeating the same task.

\section{ACKNOWLEDGEMENTS:}

This review is part of a project that has received funding from the European Research Council (ERC) under the European Union's Horizon 2020 research and innovation programme (Grant agreement No. 787307). Thanks to Thomas Colin for helpful comments on a previous draft of this manuscript. 


\section{REFERENCES:}

1. Ebitz, R.B. and Hayden, B.Y. (2016) Dorsal anterior cingulate cortex: A Rorschach test for cognitive neuroscience. Nat. Neurosci. 19, 1278-1279.

2. Silvetti, M. et al. (2014) From conflict management to reward-based decision making: Actors and critics in primate medial frontal cortex. Neurosci. Biobehav. Rev. 46, 44-57.

3. Vassena, E. et al. (2017). Computational models of anterior cingulate cortex: At the crossroads between prediction and effort. Front. Neurosci. 11, Article 316.

4. Botvinick, M. and Weinstein, A. (2014) Model-based hierarchical reinforcement learning and human action control. Philos. Trans. R. Soc. Lond. B Biol. Sci. 369, 20130480.

5. Sutton, R.S. and Barto, A.G. (2020) Reinforcement Learning: An Introduction, The MIT Press.

6. Posner, M.I. and DiGirolamo, G.J. (1998) Executive attention: Conflict, target detection, and cognitive control. In The Attentive Brain (Parasuraman, R., ed), pp. 401-423, The MIT Press.

7. Botvinick, M.M. et al. (2001) Conflict monitoring and cognitive control. Psychol. Rev. 108, 624-52.

8. Rushworth, M.F. et al. (2004) Action sets and decisions in the medial frontal cortex. Trends Cogn. Sci. 8, 410-417.

9. Holroyd, C.B. and Coles, M.G.H. (2002) The neural basis of human error processing: Reinforcement learning, dopamine, and the error-related negativity. Psychol. Rev. 109, 679-709.

10. Verbeke, P. and Verguts, T. (2019) Learning to synchronize: How biological agents can couple neural task modules for dealing with the stability-plasticity dilemma. PLoS Comput. Biol. 15, e10066o4.

11. Powell, N.J. and Redish, A.D. (2016) Representational changes of latent strategies in rat medial prefrontal cortex precede changes in behaviour. Nat. Commun. 7, 12830.

12. Sarafyazd, M. and Jazayeri, M. (2019) Hierarchical reasoning by neural circuits in the frontal cortex. Science 364, eaav8911.

13. Duverne, S. and Koechlin, E. (2017) Rewards and cognitive control in the human prefrontal cortex. Cereb. Cortex 27, 5024-5039.

14. Minxha, J. et al. (2020) Flexible recruitment of memory-based choice representations by the human medial frontal cortex. Science 368, eaba3313.

15. Holroyd, C.B. and McClure, S.M. (2015) Hierarchical control over effortful behavior by rodent medial frontal cortex: A computational model. Psychol. Rev. 122, 54-83.

16. Balaguer, J. et al. (2016) Neural mechanisms of hierarchical planning in a virtual subway network. Neuron 90, 893-903.

17. Shahnazian, D. et al. (2018) Electrophysiological responses of medial prefrontal cortex to feedback at different levels of hierarchy. Neuroimage 183, 121-131.

18. Ribas-Fernandes, J.J.F. et al. (2019) Subgoal- and goal-related reward prediction errors in medial prefrontal cortex. J. Cogn. Neurosci. 31, 8-23.

19. Kolling, N. et al. (2016) Value, search, persistence and model updating in anterior cingulate cortex. Nat. Neurosci. 19, 1280-1285.

20. Miller, K.J. and Venditto, S.J.C. (2021) Multi-step planning in the brain. Curr. Opin. Behav. Sci. 38, 29-39.

21. Alexander, W.H. and Brown, J.W. (2015) Hierarchical error representation: A computational model of anterior cingulate and dorsolateral prefrontal cortex. Neural Comput. 27, 2354-2410.

22. Shahnazian, D. and Holroyd, C.B. (2018) Distributed representations of action sequences in anterior cingulate cortex: A recurrent neural network approach. Psychon. Bull. Rev. 25, 302- 
321.

23. Yoo, S.B.M. et al. (2020) The neural basis of predictive pursuit. Nat. Neurosci. 23, 252-259.

24. Juechems, K. and Summerfield, C. (2019) Where does value come from? Trends Cogn. Sci. 23, 836850.

25. Doll, B.B. et al. (2015) Model-based choices involve prospective neural activity. Nat. Neurosci. 18, 767-772.

26. Alexander, W.H. and Brown, J.W. (2019) The role of the anterior cingulate cortex in prediction error and signaling surprise. Top. Cogn. Sci. 11, 119-135.

27. Momennejad, I. et al. (2018) Offline replay supports planning in human reinforcement learning. Elife 7, e32548.

28. Kolling, N. et al. (2018) Prospection, perseverance, and insight in sequential behavior. Neuron 99, 1069-1082.

29. Simon, D.A. and Daw, N.D. (2011) Neural correlates of forward planning in a spatial decision task in humans. J. Neurosci. 31, 5526-5539.

30. Kaplan, R. et al. (2017) The neural representation of prospective choice during spatial planning and decisions. PLoS Biol. 15, e1002588.

31. Javadi, A.H. et al. (2019) Backtracking during navigation is correlated with enhanced anterior cingulate activity and suppression of alpha oscillations and the 'default-mode' network. Proc. Biol. Sci. 286, 20191016.

32. Mashhoori, A. et al. (2018) Rat anterior cingulate cortex recalls features of remote reward locations after disfavoured reinforcements. Elife 7, e29793.

33. Akam, T. et al. (2020) Anterior cingulate cortex represents action-state predictions and causally mediates model-based reinforcement learning in a two-step decision task. bioRxiv doi: 10.1101/126292.

34. Shenhav, A. et al. (2017) Toward a rational and mechanistic account of mental effort. Annu. Rev. Neurosci. 40, 99-124.

35. Lieder, F. et al. (2018) Rational metareasoning and the plasticity of cognitive control. PLoS Comput. Biol. 14, e1006043.

36. Silvetti, M. et al. (2018) Dorsal anterior cingulate-brainstem ensemble as a reinforcement metalearner. PLoS Comput. Biol. 14, e1006370.

37. Kool, W. et al. (2017) Cost-benefit arbitration between multiple reinforcement-learning systems. Psychol. Sci. 28, 1321-1333.

38. Holroyd, C.B. (2016) The waste disposal problem of effortful control. In Motivation and Cognitive Control (Braver, T., ed), pp. 235-26o, Psychology Press.

39. Hayden, B.Y. (2019) Why has evolution not selected for perfect self-control? Philos. Trans. R. Soc. Lond. B. Biol. Sci. 374, 20180139.

40. Finley, A.J. et al. (2019) Sweet nothings: No effects of self-control exertion on blood-glucose levels. Soc. Psychol. 50, 322-331.

41. Boureau, Y.L. et al. (2015) Deciding how to decide: Self-control and meta-decision making. Trends Cogn. Sci. 19, 700-710.

42. Botvinick, M.M. and Cohen, J.D. (2014) The computational and neural basis of cognitive control: Charted territory and new frontiers. Cogn. Sci. 38, 1249-1285.

43. Abrahamse, E. et al. (2016) Grounding cognitive control in associative learning. Psychol. Bull. 142, 693-728.

44. Badre, D. and Nee, D.E. (2018) Frontal cortex and the hierarchical control of behavior. Trends 
Cogn. Sci. 22, 170-188.

45. Holroyd, C.B. and Yeung, N. (2012) Motivation of extended behaviors by anterior cingulate cortex. Trends Cogn. Sci. 16, 122-128.

46. Juechems, K. et al. (2019) A network for computing value equilibrium in the human medial prefrontal cortex. Neuron 101, 977-987.

47. Shenhav, A. et al. (2018) Dissociable neural mechanisms track evidence accumulation for selection of attention versus action. Nat. Commun. 9, 2485.

48. Domenech, P. et al. (2020) Neural mechanisms resolving exploitation-exploration dilemmas in the medial prefrontal cortex. Science 369, eabbo184.

49. Fries, P. (2015) Rhythms for cognition: Communication through coherence. Neuron 88, 220-235.

50. Yamashita, Y. and Tani, J. (2008) Emergence of functional hierarchy in a multiple timescale neural network model: A humanoid robot experiment. PLoS Comput. Biol. 4, e1000220.

51. Buzsáki, G. et al. (2012) The origin of extracellular fields and currents--EEG, ECoG, LFP and spikes. Nat. Rev. Neurosci. 13, 407-420.

52. Verguts, T. (2017) Binding by random bursts: A computational model of cognitive control. J. Cogn. Neurosci. 29, 1103-1118.

53. Holroyd, C.B. and Umemoto, A. (2016) The research domain criteria framework: The case for anterior cingulate cortex. Neurosci. Biobehav. Rev. 71, 418-443.

54. Shine, J.M. (2019) Neuromodulatory influences on integration and segregation in the brain. Trends Cogn. Sci. 23, 572-583.

55. Dayan, P. and Yu, A.J. (2006) Phasic norepinephrine: A neural interrupt signal for unexpected events. Network 17, 335-350.

56. Karlsson, M.P. et al. (2012) Network resets in medial prefrontal cortex mark the onset of behavioral uncertainty. Science 338, 135-139.

57. Rogers, T.T. and McClelland, J.L. (2014) Parallel Distributed Processing at 25: Further explorations in the microstructure of cognition. Cogn. Sci. 38, 1024-1077.

https://doi.org/10.1111/cogs.12148

58. Saxena, S. and Cunningham, J.P. (2019) Towards the neural population doctrine. Curr. Opin. Neurobiol. 55, 103-111.

59. Heilbronner, S.R and Hayden, B.Y. (2016) Dorsal anterior cingulate cortex: A bottom-up view. Annu. Rev. Neurosci. 39, 149-170.

6o. Ma, L. et al. (2016) A quantitative analysis of context-dependent remapping of medial frontal cortex neurons and ensembles. J. Neurosci. 36, 8258-8272.

61. Lapish, C.C. et al. (2015) Amphetamine exerts dose-dependent changes in prefrontal cortex attractor dynamics during working memory. J. Neurosci. 35, 10172-10187.

62. Enel, P. et al. (2016). Reservoir computing properties of neural dynamics in prefrontal cortex. PLoS Comput. Biol. 12, e1004967.

63. Holroyd, C.B. et al. (2018) Human midcingulate cortex encodes distributed representations of task progress. Proc. Natl. Acad. Sci. U. S. A. 115, 6398-6403.

64. Ebitz, R.B. et al. (2020) Human dorsal anterior cingulate neurons signal conflict by amplifying task-relevant information. bioRxiv doi: 10.1101/2020.03.14.991745.

65. Ju, H. and Bassett, D.S. (2020) Dynamic representations in networked neural systems. Nat. Neurosci. 23, 908-917.

66. Vyas, S. et al. (2020) Computation through neural population dynamics. Annu. Rev. Neurosci. 43, 249-275. 
67. Yang, G.R and Wang, X.J. (2020) Artificial neural networks for neuroscientists: A primer. Neuron 107, 1048-1070.

68. Rabinovich, M.I. et al. (2015) Dynamical bridge between brain and mind. Trends Cogn. Sci. 19, 453-461.

69. Remington, E.D. et al. (2018) Flexible sensorimotor computations through rapid reconfiguration of cortical dynamics. Neuron 98, 1005-1019.

70. Konidaris, G. (2019) On the necessity of abstraction. Curr. Opin. Behav. Sci. 29, 1-7.

71. Schmidhuber, J. (2015) Deep learning in neural networks: An overview. Neural Netw. 61, 85-117.

72. Lillicrap, T.P. et al. (2020) Backpropagation and the brain. Nat. Rev. Neurosci. 21, 335-346.

73. Wang, J.X. et al. (2018) Prefrontal cortex as a meta-reinforcement learning system. Nat. Neurosci. 21, 860-868.

74. Botvinick, M. et al. (2019) Reinforcement learning, fast and slow. Trends Cogn. Sci. 23, 408-422.

75. Westbrook, A. and Braver, T.S. (2016) Dopamine does double duty in motivating cognitive effort. Neuron 89, 695-710.

76. Frobose, M.I. and Cools, R. (2018) Chemical neuromodulation of cognitive control avoidance. Curr. Opin. Behav. Sci. 22, 121-127.

77. Gilboa, A. and Marlatte, H. (2017) Neurobiology of schemas and schema-mediated memory. Trends Cogn. Sci. 21, 618-631.

78. Baldassano, C. et al. (2018) Representation of real-world event schemas during narrative perception. J. Neurosci. 38, 9689-9699.

79. Tomov, M.S. et al. (2020) Discovery of hierarchical representations for efficient planning. PLoS Comput. Biol. 16, e1007594.

8o. Huys, Q.J. et al. (2015) Interplay of approximate planning strategies. Proc. Natl. Acad. Sci. U. S. A. 112, 3098-3103.

81. Tartaglia, E.M. et al. (2017) What to choose next? A paradigm for testing human sequential decision making. Front. Psychol. 8, 312.

82. Le Heron, C. et al. (2019) Brain mechanisms underlying apathy. J. Neurol. Neurosurg. Psychiatry 90, 302-312.

83. Behrens, T.E.J. et al. (2018) What is a cognitive map? Organizing knowledge for flexible behavior. Neuron 100, 490-509.

84. Gershman, S.J. and Daw, N.D. (2017) Reinforcement learning and episodic memory in humans and animals: An integrative framework. Annu. Rev. Psychol. 68, 101-128.

85. Schmidt, B. et al. (2019) Disrupting the medial prefrontal cortex alters hippocampal sequences during deliberative decision making. J. Neurophysiol. 121, 1981-20oo.

86. Baraduc, P. et al. (2019) Schema cells in the macaque hippocampus. Science 363, 635-639.

87. Yang, G.R. et al. (2019) Task representations in neural networks trained to perform many cognitive tasks. Nat. Neurosci. 22, 297-306.

88. Niv, Y. (2019) Learning task-state representations. Nat. Neurosci. 22, 1544-1553.

89. Jahn, A. et al. (2016) Distinct regions within medial prefrontal cortex process pain and cognition. J. Neurosci. 36, 12385-12392.

90. Laubach, M. et al. (2018) What, if anything, is rodent prefrontal cortex? eNeuro 5, e0315-18.2018.

91. van Heukelum, S. et al. (2020) Where is cingulate cortex? A cross-species view. Trends Neurosci. 43, 285-299.

92. Procyk, E. et al. (2016) Midcingulate motor map and feedback detection: Converging data from humans and monkeys. Cereb. Cortex 26, 467-476. 
93. Elsayed, G.F. and Cunningham, J.P. (2017) Structure in neural population recordings: An expected byproduct of simpler phenomena? Nat. Neurosci. 20, 1310-1318.

94. Cunningham, J.P. and Yu, B.M. (2014) Dimensionality reduction for large-scale neural recordings. Nat. Neurosci. 17, 1500-1509.

95. Richards, B.A. et al. (2019) A deep learning framework for neuroscience. Nat. Neurosci. 22, 17611770.

96. Botvinick, M. and Plaut, D.C. (2004) Doing without schema hierarchies: A recurrent connectionist approach to normal and impaired routine sequential action. Psychol. Rev. 111, 395-429.

97. Hassabis, D. et al. (2017) Neuroscience-inspired artificial intelligence. Neuron 95, 245-258.

98. Madhav, M.S. and Cowan, N.J. (2020) The synergy between neuroscience and control theory: The nervous system as inspiration for hard control challenges. Annu. Rev. Control Robot. Auton. Syst. 3, 243-267.

99. Egner, T., ed (2017) The Wiley Handbook of Cognitive Control, Wiley-Blackwell.

10o. Lake, B. M. et al. (2017) Building machines that learn and think like people. Behav. Brain Sci. 40, e253.

101. Marblestone, A.H. et al. (2016) Toward an integration of deep learning and neuroscience. Front. Comput. Neurosci. 10, Article 94.

102. Cooper, R.P. (2017) Deep-learning networks and the functional architecture of executive control. Behav. Brain Sci. 40, e261.

103. Russin, J. et al. (2020) Deep-learning needs a prefrontal cortex. Bridging AI and Cognitive Science: International Conference on Learning Representations 2020.

104. Hamrick, J.B. (2019) Analogues of mental simulation and imagination in deep learning. Curr. Opin. Behav. Sci. 29, 8-16.

105. Collins, A.G.E. and Cockburn, J. (2020) Beyond dichotomies in reinforcement learning. Nat. Rev. Neurosci. 21, 576-586.

106. Silver, D. et al. (2018) A general reinforcement learning algorithm that masters chess, shogi, and Go through self-play. Science 362, 1140-1144.

107. Momennejad, I. (2020) Learning structures: Predictive representations, replay, and generalization. Curr. Opin. Behav. Sci. 32, 155-166.

108. Barreto, A. et al. (2020) Fast reinforcement learning with generalized policy updates. Proc. Natl. Acad. Sci. U. S. A. 117, 30079-30087.

109. Machado, M.C. et al. (2018) Eigenoption discovery through the deep successor representation. arXiv: $1710.11089 v 3$.

110. Harb, J. et al. (2018) When waiting is not an option: Learning options with a deliberation cost. arXiv: 1709.04571.

111. Fine, J.M. et al. (2020) Computational neural mechanisms of goal-directed planning and problem solving. Comput. Brain Behav. 3, 472-493.

112. Joo, H.R. and Frank, L.M. (2018) The hippocampal sharp wave-ripple in memory retrieval for immediate use and consolidation. Nat. Rev. Neurosci. 19, 744-757.

113. Kumaran, D. et al. (2016) What learning systems do intelligent agents need? Complementary learning systems theory updated. Trends Cogn. Sci. 20, 512-534.

114. Gershman, S.J. et al. (2014) Retrospective revaluation in sequential decision making: A tale of two systems. J. Exp. Psychol. Gen. 143, 182-194.

115. Deuker, L. et al. (2013) Memory consolidation by replay of stimulus-specific neural activity. J. Neurosci. 33, 19373-19383. 
116. Staresina, B.P. et al. (2013) Awake reactivation predicts memory in humans. Proc. Natl. Acad. Sci. U. S. A. 110, 21159-21164.

117. Schuck, N.W. and Niv, Y. (2019) Sequential replay of nonspatial task states in the human hippocampus. Science 364, eaaw5181.

118. Jadhav, S.P. et al. (2016) Coordinated excitation and inhibition of prefrontal ensembles during awake hippocampal sharp-wave ripple events. Neuron 90, 113-127.

119. Kaefer, K. et al. (2020) Replay of behavioral sequences in the medial prefrontal cortex during rule switching. Neuron 106, 154-165.

120. Shin, J.D. et al. (2019) Dynamics of awake hippocampal-prefrontal replay for spatial learning and memory-guided decision making. Neuron 104, 1110-1125. 


\section{FIGURE LEGENDS}

\section{FIGURE 1: Distributed Representations in Anterior Cingulate Cortex During Sequence}

Execution. (A) Trajectories of an anterior cingulate cortex (ACC) neural ensemble recorded from a rat performing a delayed-spatial win shift task. State space dimensions were derived using a bespoke multivariate decomposition method. Arrow color and length indicate state phase and velocity, respectively, associated with different stages of trial execution (see legend). Adapted with permission from (AWPF) [61]. (B) Trajectories summarizing the ensemble network activity of monkey ACC (left) and recurrent neural network (RNN) hidden units (right) during performance of a trial-and-error learning task. State space dimensions were derived using principal components analysis. Line symbols and colors indicate successive events within a trial and successive trials within a sequence, respectively (see legend). Arrows indicate direction of flow. INCOR: incorrect trial. COR: correct trial. AWPF [62]. (C) Trajectories summarizing patterns of the fMRI BOLD response in human ACC (left) and RNN hidden unit activity (right) during a simulated coffee-tea making task. State space dimensions were derived using multidimensional scaling analysis. Line colors and styles indicate task type and within-sequence order, respectively; line symbols indicate event categories (see legend) that are labeled according to state space region (B: Begin. A: Add. S: Stir. E: End.). Data re-analyzed from [63]. (D) Illustration of a stable heteroclinical channel, a mathematical object that represents hierarchical action sequences as successive metastable states. Blue and green trajectories indicate the dynamics within and between sequences, respectively. Arrows indicate direction of flow. AWPF [68]. 
FIGURE 2, Key Figure: Anterior Cingulate Cortex at the Wheel of Hierarchical Planning. Different brain modules (colored boxes) make distinct contributions to a neural network for hierarchical planning. Hippocampus (pink box) supports the construction of world models that are gradually transferred and consolidated in cortex. Planning is mediated via thalamo-cortical-striatal loops (not shown) that link parallel structures in lateral prefrontal cortex (LPC), medial prefrontal cortex (and anterior cingulate cortex; ACC), and the basal ganglia. The ACC utilizes world models to predict the value of and select plans for execution. This function is mediated by a gradient of hierarchically-organized modules (blue boxes connected by double-arrow), with rostral parts (including neighboring areas in medial orbitofrontal cortex, mOFC) concerned with abstract representations such as "goals", "tasks", and action "schemas", and caudal-dorsal parts (including the pre-supplementary motor area; pre-SMA) concerned with concrete representations such as "lowerlevel goals" and "actions". These functions are encoded within each module as distributed representations (network grids) and bound together between modules with neural oscillations (waves). Once selected, the LPC and the basal ganglia (yellow boxes) implement the details of the plan. ACC monitors plan execution, weighing the predicted benefits against the incurred costs, supporting plans as appropriate by increasing module binding (thicker waves) that incurs larger costs (price tag). Neuromodulators including dopamine (green box) and norepinephrine (not shown) carry reward signals (currency) and other task variable to ACC (and elsewhere; connections not shown), where they regulate the stability of distributed representations and between-module binding. See main text for details. 


\section{OUTSTANDING QUESTIONS BOX:}

- Is the limitation on control imposed by ACC computationally useful (a feature) or biologically necessary (a bug)?

- If the limitation on control constitutes a feature, not a bug, then should future robots exploit this limitation, occasionally giving up on tasks that they are otherwise capable of achieving?

- How does the ACC identify subgoals in complicated tasks? How are these integrated across hierarchical levels into schemas?

- $\quad$ Can we build models of real-world planning that are useful to both cognitive neuroscience and Al?

- $\quad$ Conventional computational models of ACC mostly use either tabular representations associated with standard RL, or distributed representations associated with ANNs. How can the two approaches be better integrated? Can these be augmented to encode compositional representations associated with symbolic Al?

- $\quad$ The majority of ACC studies establish correlational rather than causal relationships. How can we find causal evidence that ACC supports HMB-HRL in humans?

- Can the temporal evolutions of task representations in ACC be described using nonlinear dynamical equations? 


\section{HIGHLIGHTS BOX:}

- Three core principles of anterior cingulate cortex (ACC) function are reviewed: hierarchy, world-models, and cost.

- $\quad$ Four neural considerations regarding the biophysical implementation of these principles are discussed: modularity, binding, encoding, and learning and regulation.

- $\quad$ These observations suggest that ACC motivates hierarchical model-based hierarchical reinforcement learning. 


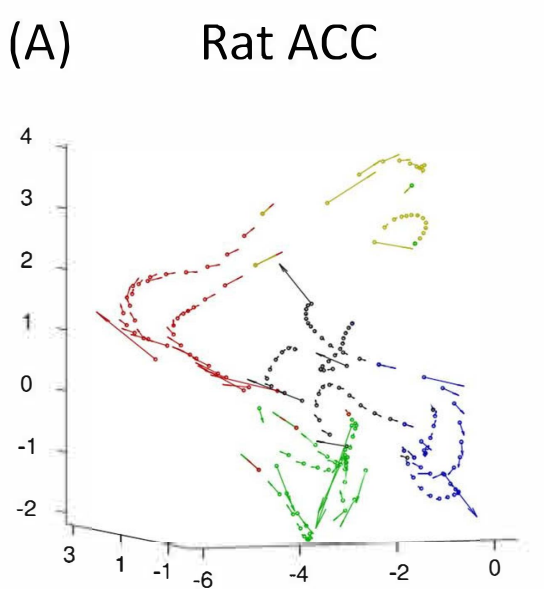

- Training Choice • Training Reward - Incorrect Choice - Test Choice • Test Reward
(B) Monkey ACC

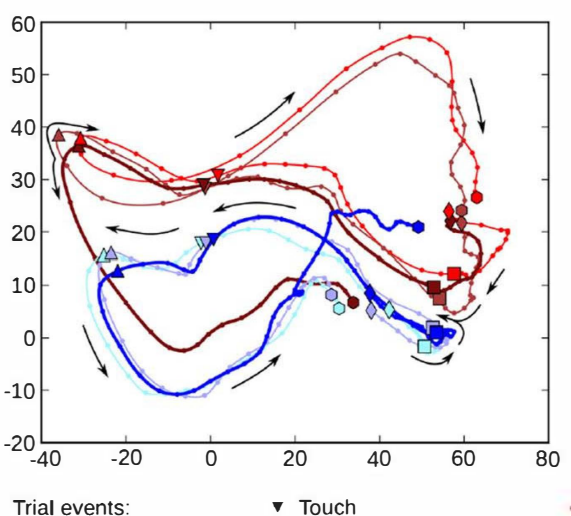

- Trial start (lever touch) \ Feedback

- Targets appear - Trial end (next lever touch)
Recurrent Network

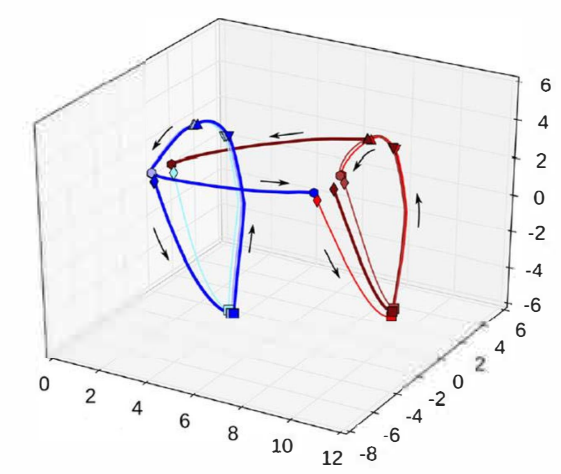

INCOR2 $\}$ Search phase - COR3 3 COepeat phase
(C) Human ACC

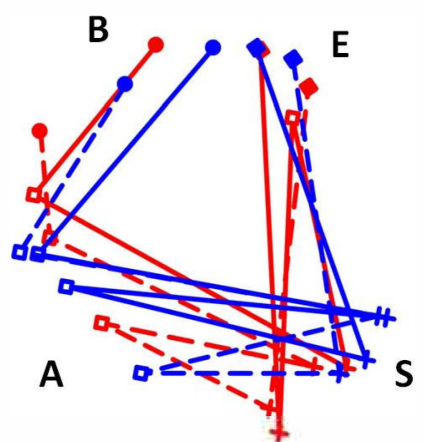

- Choose drink (tea, coffee)

Add ingredient (sugar, cream, water)

$X$ Stir

Serve drink (tea, coffee)
Recurrent Network

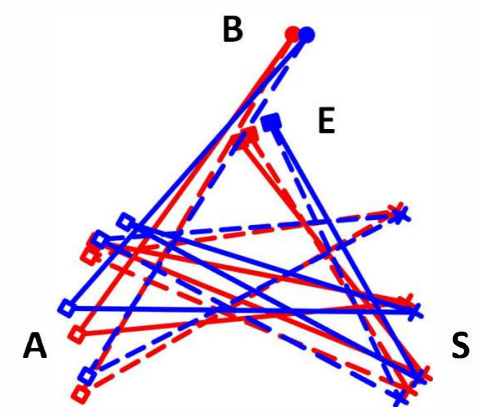

Make tea, add water first Make tea, add sugar first

Make coffee, add water first Make coffee, add cream first
(D) Dynamics Model

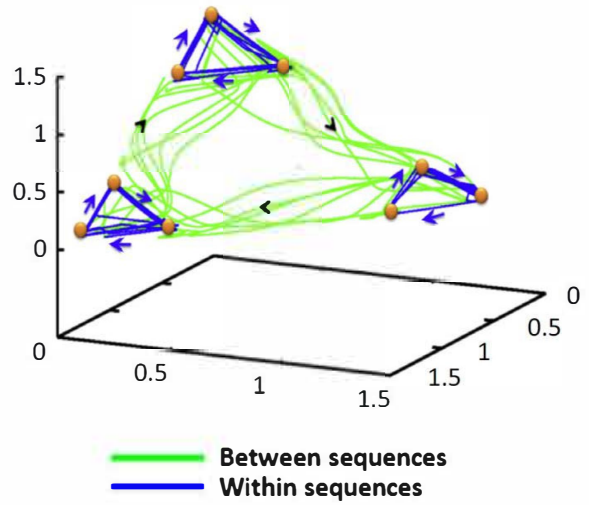




\section{lateral prefrontal basal ganglia}

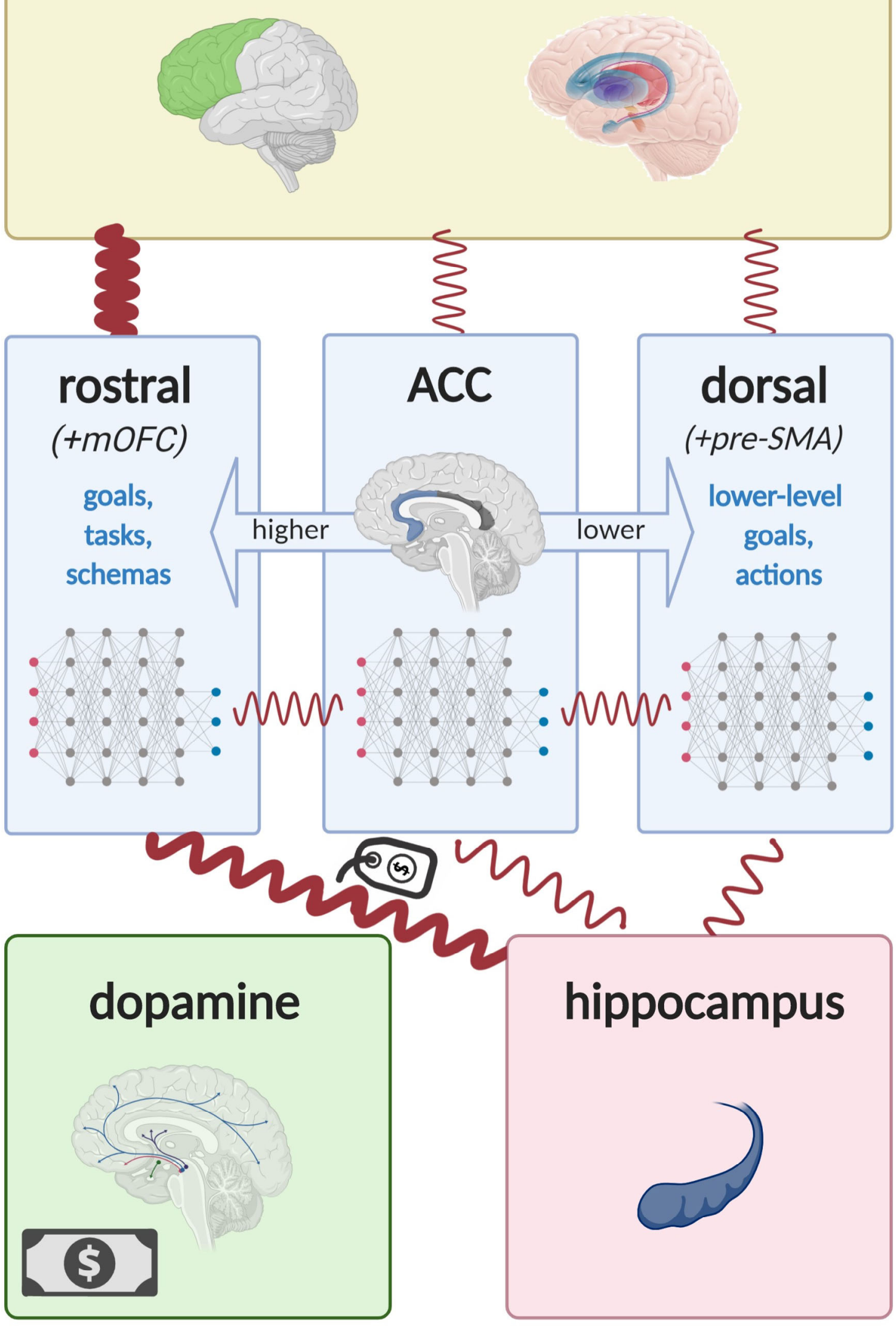

Plan execution
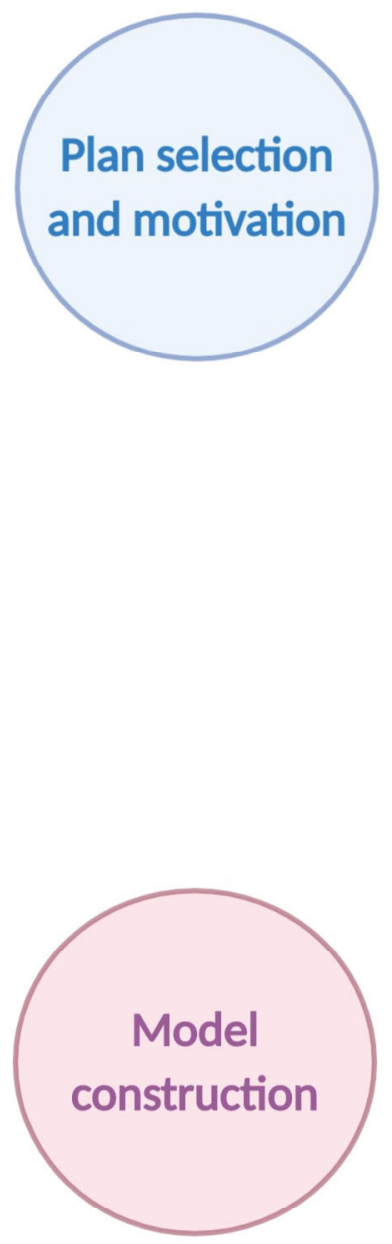

Figure 2 
(A)

(B) pre-SMA

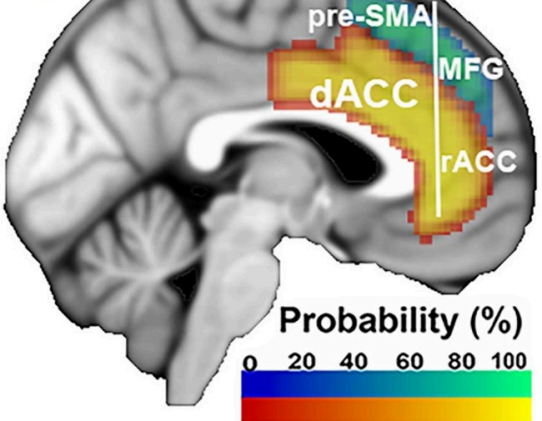

(C)

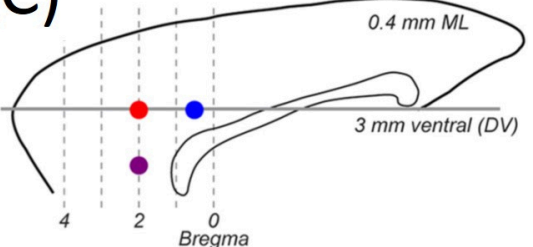

Figure Box 1

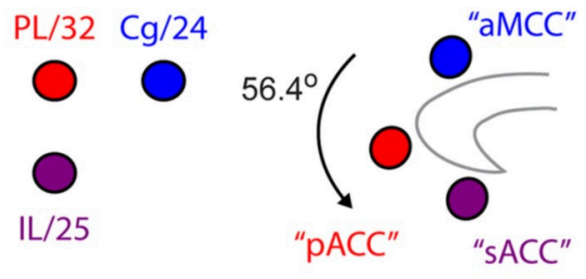



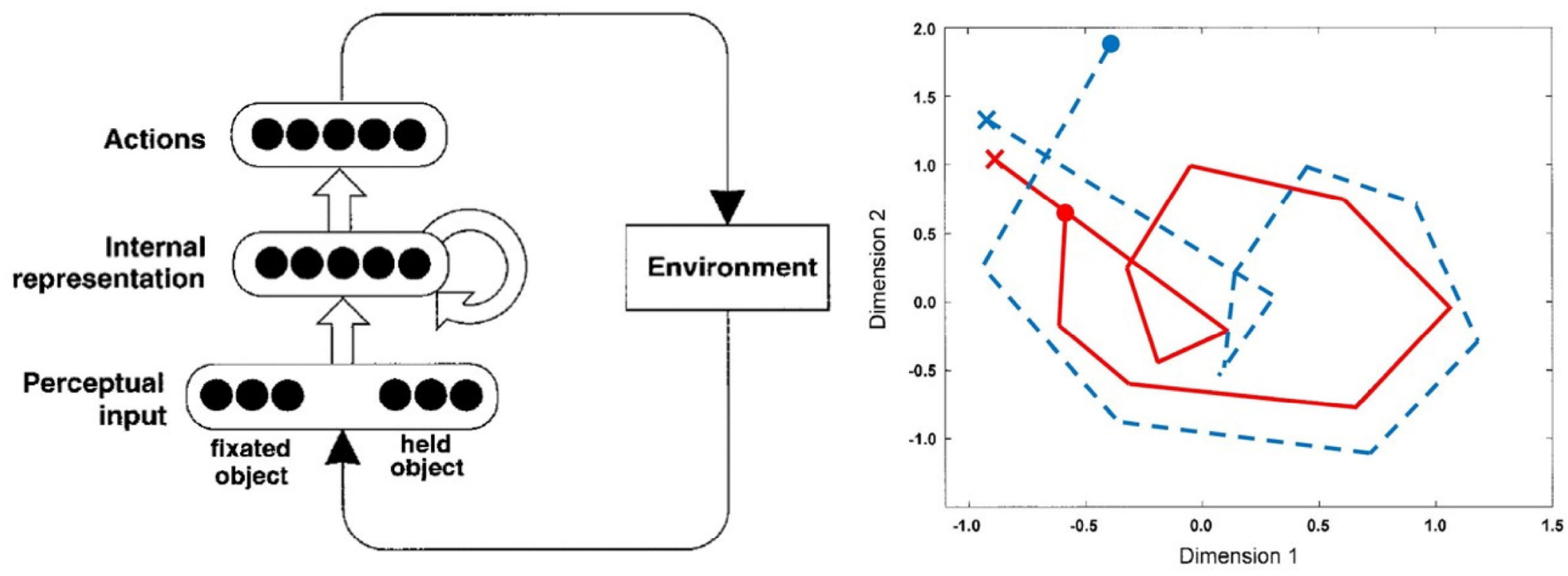